\title{
Light-driven self-assembly of cyanostilbene derivative with reversible chirality in aqueous media
}

\author{
Xiaoxuan Zeng, Yue $\mathrm{Wu}^{*}$, Lin Zou, Xingwang Liu, Xin Qi and Zhen-Qiang Yu*
}

Chirality, which is one of the defining features of biological molecules, plays a critical role in many important life processes. Controlled supramolecular assembly of biomolecules into functional structures with low structural fluidity, e.g., cytoskeleton filaments [1], flagellar filaments of bacteria [2], and high structural fluidity (e.g., cell membranes [3]), serves key roles in the correct functioning of biological processes. Inspired by natural supramolecular assembly, a large number of discrete two- and three-dimensional chiral polygons with well-defined shapes have been successfully prepared through self-assembly [4-8]. In particular, chiral amplification by self-assembly of chiral building blocks into artificial nanostructures shows great promise in materials science [9-12]. There are, however, few examples that display chiral switching in response to external signals, in contrast to the well-controlled tunability of chirality in living systems $[13,14]$. Stimuli-responsive strategies to control the assembly system using switchable chiral signals, especially in aqueous media, would thus be very valuable.

Light is a perfect external trigger to stimulate self-assembly since photon input is not only clean and waste-free, but also offers precise spatiotemporal control [15-26]. Photon-triggered chiroptical switching in molecules and materials is attracting particular interest because the simultaneous realization of both optical and chiral signals can add extra information density [2735]. With this in mind, we envisioned a new photochromism that combines chirality and amphiphilicity. Herein, we describe a rational molecular design strategy to prepare trans-CS by combining a photochromic unit (cyanostilbene), a hydrophilic unit (sulfonate), and a hydrophobic chiral moiety (cholesterol) through flexible spacers (Scheme S1, Figs S1-S8 and Fig. 1). The chiral and amphiphilic trans-CS showed photoswitchable chirality, which accompanied self-assembly or disassembly in aqueous media. This newly designed material showed the following extraordinary features: (i) self-assembly into spherical aggregates to create circular dichroism (CD) signals with good reversibility, (ii) $90 \%$ conversion yield from trans-CS to cis-CS upon irradiation at $365 \mathrm{~nm}$, and (iii) excellent thermal stability in water. Importantly, this approach provides a general route to the selfassembly of water-soluble, chiral, and photoswitchable amphiphiles.

Because of the presence of the cyanostilbene moiety, trans-CS is expected to undergo trans/cis isomerization when irradiated with ultraviolet (UV) light. ${ }^{1} \mathrm{H}$ nuclear magnetic resonance (NMR) spectra recorded during the UV-light-induced transformation can be used to follow the trans-CS to cis-CS process
(Fig. S9). The ratio of trans-CS to cis-CS could be calculated using the integration ratio of the original and new signals. After irradiation with $365 \mathrm{~nm}$ light for $5 \mathrm{~min}$, the trans/cis ratio in solution was 9:1. The amount of trans-CS gradually decreased with increasing irradiation time and the trans/cis ratio stabilized at 1:9 after irradiation for $40 \mathrm{~min}$. The high photoconversion from trans-CS to cis-CS (90\% after $40 \mathrm{~min}$ ) indicates the high efficiency of the photoisomerization reaction.

When the concentration of trans-CS in aqueous solution was increased from 0.01 to $1 \mathrm{mmol} \mathrm{L}^{-1}$, an absorption peak at $336 \mathrm{~nm}$ and a CD peak at $361 \mathrm{~nm}$ appeared synchronously (Fig. 2a, b). The inset in Fig. 2a shows the absorbance at $336 \mathrm{~nm}$ increased linearly with different slopes when the concentration was lower or higher than $0.1 \mathrm{mmol} \mathrm{L}^{-1}$. The change in slope indicates the sample self-assembled at the appropriate concentration of $0.1 \mathrm{mmol} \mathrm{L}^{-1}$. Meanwhile, the concentration dependence of CD intensity in the inset of Fig. $2 b$ also shows the self-assembly concentration was $0.1 \mathrm{mmol} \mathrm{L}^{-1}$ in water. Thus, we selected $0.1 \mathrm{mmol} \mathrm{L}^{-1}$ trans-CS to investigate its selfassembly behavior. Meanwhile, the reference compound CS-OH without cholesterol group shows no CD signal, confirming that the chirality of trans-CS was originated from the cholesteric moiety (Fig. S10). Trans-CS, which absorbs strongly at $336 \mathrm{~nm}$ (Fig. 2c) in water, can be photoconverted to cis-CS upon irradiation at $365 \mathrm{~nm}\left(10 \mathrm{~mW} \mathrm{~cm}^{-2}\right)$. The resultant cis-isomer has an absorption band at $237 \mathrm{~nm}$, and the appearance of this band is a characteristic indicator of transformation from a trans-isomer to a cis-isomer. The photostationary state (PSS) shows weak absorption at $336 \mathrm{~nm}$, indicating majority of trans-isomer isomerized into cis-isomer (Fig. 2a). Conversely, irradiation of photochemically generated cis-CS with $254 \mathrm{~nm}$ light mostly converts it back to the trans-isomer (90\%).

The trans-CS/cis-CS system also exhibits good fatigue resistance. This is exemplified in Fig. 2d, which shows five rounds of back-and-forth shuttling between trans-CS and cis-CS, brought about by alternating irradiation with 365 or $254 \mathrm{~nm}$ light. The absorbance repeatedly oscillates between the minimum and maximum values, which correspond to the absorption values of trans-CS and cis-CS, respectively. Synchronously, the CD signals of the trans/cis-CS system also display oscillations (Fig. 2e), indicating that the optical chirality is strongly correlated with the isomeric structure, although the chiral moiety (cholesterol) is unchanged. Furthermore, the photoisomerization of trans-CS can be shown in the characterization of fluorescence performance (Fig. S11). Unlike many known photochromisms [36,37],

College of Chemistry and Environmental Engineering, Institute of Low-dimensional Materials Genome Initiative, Shenzhen University, Shenzhen 518071, China

* Corresponding authors (emails: wuyue@szu.edu.cn (Wu Y); zqyu@szu.edu.cn (Yu ZQ)) 

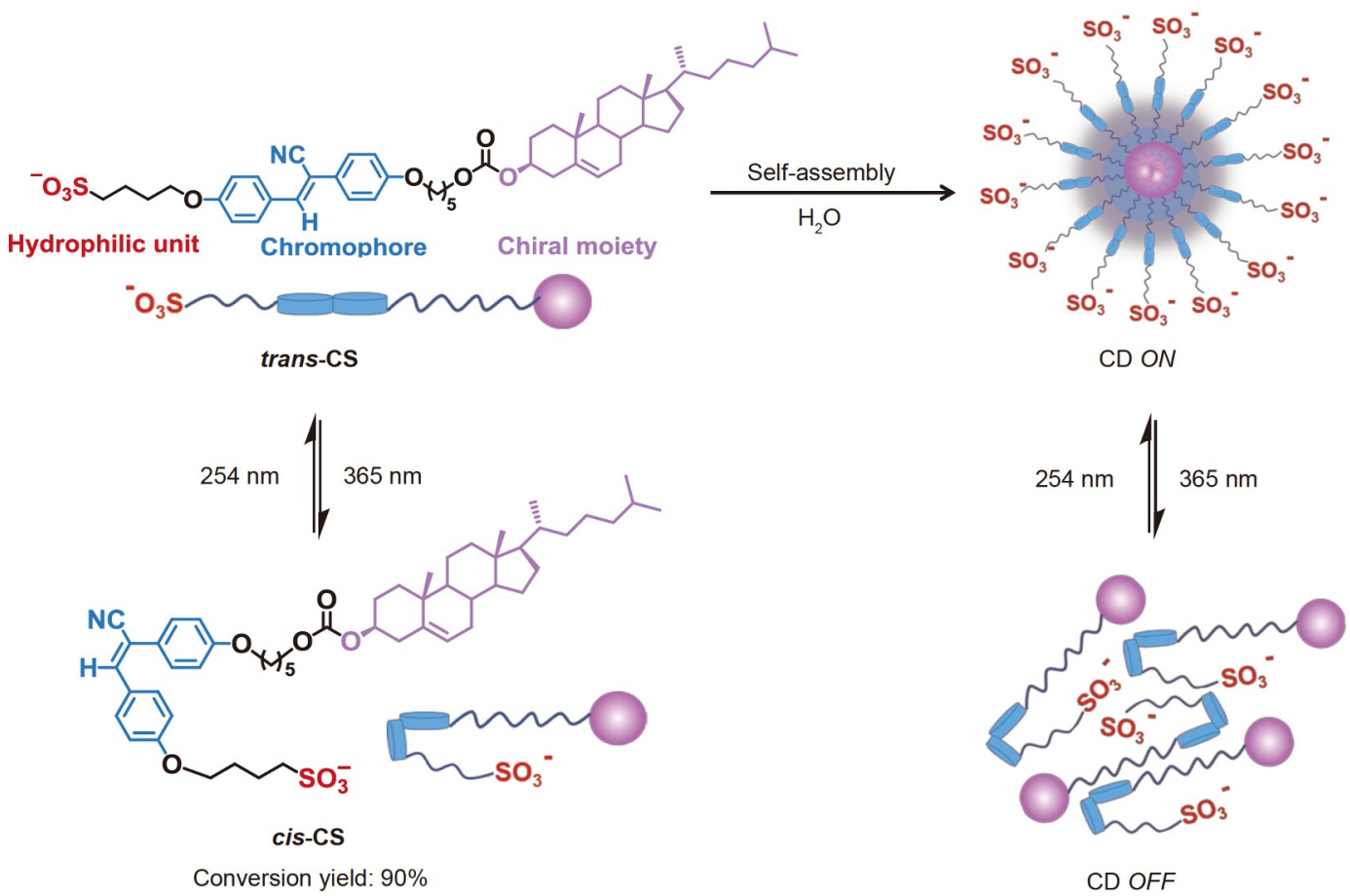

Figure 1 Reversible dual UV light shuttle of cyanostilbene between its two stable states, trans-CS and cis-CS. When self-assembled into spherical aggregates, trans-CS produces a strong CD signal, which disappears upon conversion to cis-CS by irradiation with $365 \mathrm{~nm}$ light.
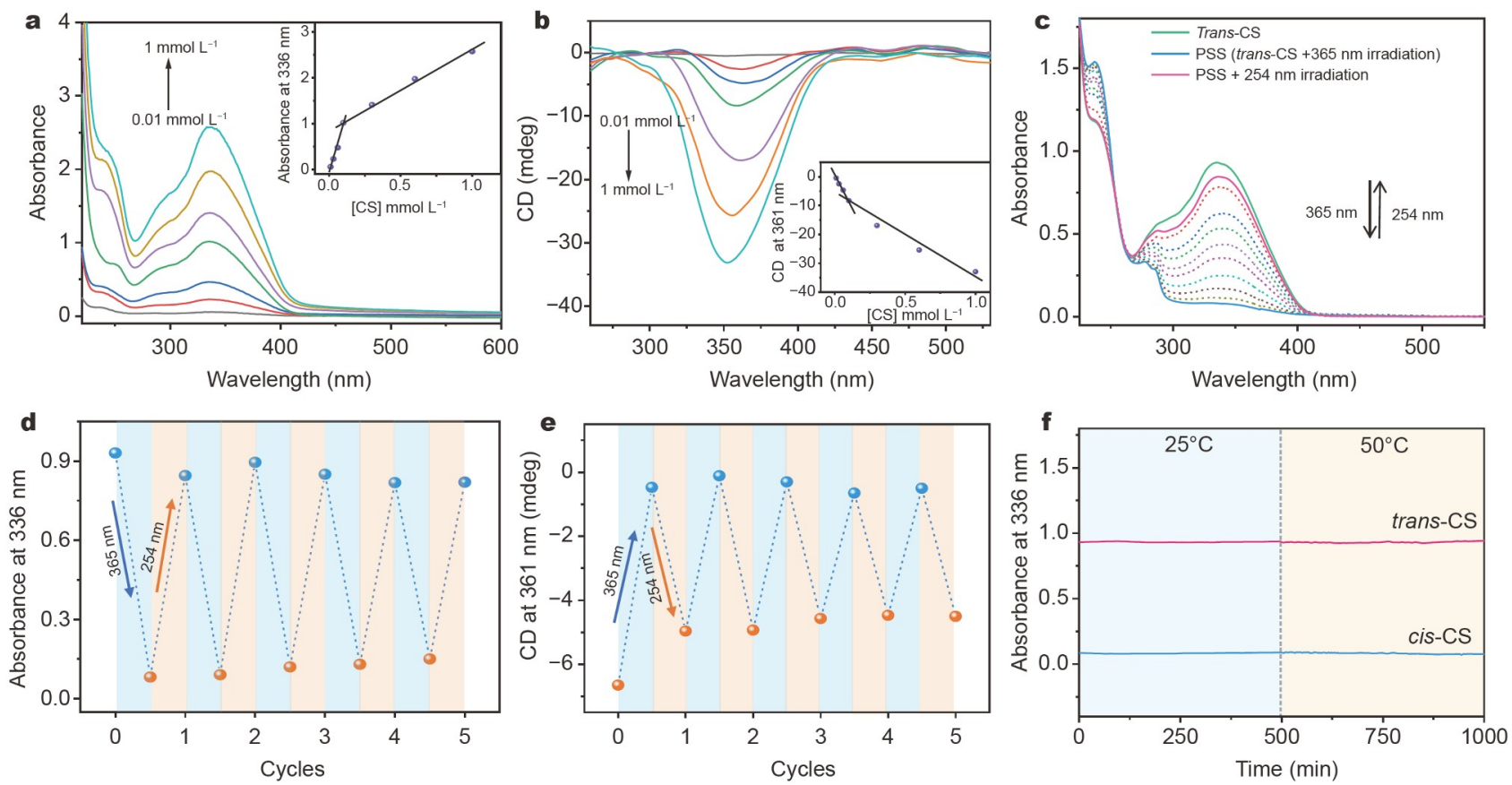

Figure 2 Absorption (a) and CD (b) showing dynamic variations in self-assembly with increasing concentration of trans-CS. Insets in (a): absorbance changes at $336 \mathrm{~nm},(\mathrm{~b})$ : CD changes at $361 \mathrm{~nm}$. (c) High conversion photoswitching between trans-CS and cis-CS upon dual UV light shuttle (0.1 mmol L ${ }^{-1}$, $\left.\mathrm{H}_{2} \mathrm{O}\right)$. Alternation between $365 \mathrm{~nm}$ light $\left(140 \mathrm{~mW} \mathrm{~cm}{ }^{-2}\right)$ for $40 \mathrm{~min}$ and $254 \mathrm{~nm}$ light $\left(10 \mathrm{~mW} \mathrm{~cm}^{-2}\right)$ for 6 h reversibly photoswitches between trans- and cisisomers in water, demonstrating good fatigue resistance and reversibility of absorption (d) and optical chirality (e) in aqueous media. (f) Absorbance of transCS and cis-CS in water monitored at $25^{\circ} \mathrm{C}$ for $500 \mathrm{~min}$, followed by heating at $50^{\circ} \mathrm{C}$ for $500 \mathrm{~min}$. No change in absorbance was observed at $336 \mathrm{~nm}$, indicating that cis-CS and trans-CS are thermally stable (bistable states).

trans-CS and cis-CS both have excellent thermal stability and do not thermally interconvert. The two isomers showed no interconversion after $500 \mathrm{~min}$ at room temperature and no change in absorption at $336 \mathrm{~nm}$ was observed after heating to $50^{\circ} \mathrm{C}$ for $500 \mathrm{~min}$ (Fig. 2f). Together, these data reveal that both trans-CS and $\boldsymbol{c i s}$-CS have excellent thermal stability (bistable states) and 
good photoswitchable properties.

We next investigated the morphologies of the self-assemblies of trans-CS and cis-CS that give rise to the different optical properties of the two isomers. Chiral trans-CS and cis-CS contain a hydrophilic sulfonate unit and a hydrophobic cholesterol moiety and are thus amphiphilic. As above mentioned, trans-CS can self-assemble to form nanoparticles. Microscopic experiments were carried out to probe the morphology and size of the resulting aggregates. Transmission electron microscopy (TEM), using an aqueous solution of trans-CS, revealed the presence of spherical micelles, with an average diameter of $140 \mathrm{~nm}$ (Fig. 3a). This size estimation was also supported by dynamic light scattering measurements, which showed a hydrodynamic diameter of around $150 \mathrm{~nm}$ (Fig. 3c). We hypothesize that, to minimize contact between hydrophobic segments and water, the cholesterol units aggregate in the interior of the aggregate and are surrounded by hydrophilic $\mathrm{SO}_{3}{ }^{-}$units, leading to the formation of micelle structures. Upon irradiation with $365 \mathrm{~nm}$ UV light and conversion of trans-CS to cis-CS, these self-assemblies are transformed into irregular nanoparticles, with a small hydrodynamic diameter of around $18 \mathrm{~nm}$ (Fig. 3b, d). Upon light-driven trans-cis isomerization, the newly generated cis-isomers disrupt the equilibrium of the selfassembled system and thus contribute to the disassembly of the original nanospheres, as well as to the formation of smaller irregular nanoparticles. These results support the notion that trans-CS self-assembles into spherical aggregates, which then disassemble into irregular fragments on the photoisomerization of trans-CS to cis-CS, indicating that the different molecular structures and packing result in different morphologies of selfassembled particles and different hydrophilicities.

In summary, we presented a design strategy for a chiral, amphiphilic, and photoswitchable molecule, using cholesterol, sulfonate, and cyanostilbene. The molecule could self-assemble to the trans-isomer in aqueous solution, which is accompanied by strong CD signals. Both trans- and cis-isomers are thermally stable, and the high conversion photoswitching between the trans- and cis-isomers with good fatigue resistance and reversibility, can be achieved using different wavelengths of UV irra-
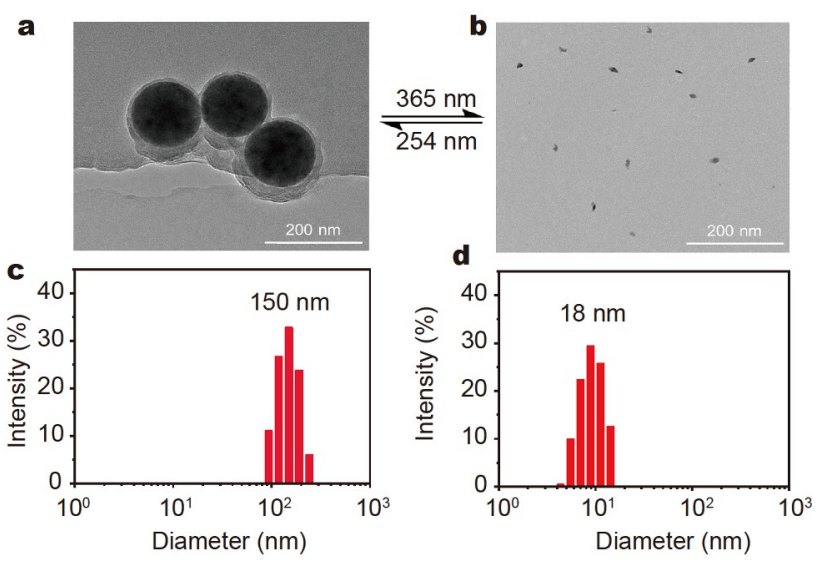

Figure 3 Self-assembly properties of trans-CS and cis-CS. (a) TEM images of spherical nanoparticles formed by trans-CS in aqueous media. (b) TEM images of irregular fragments with significantly smaller size formed by cisCS after $365 \mathrm{~nm}$ UV irradiation of trans-CS. Dynamic light scattering of nanoparticles formed by trans-CS before (c) and after (d) $365 \mathrm{~nm}$ UV irradiation in $\mathrm{H}_{2} \mathrm{O}$. diation. Furthermore, the optical chirality-CD was also modulated by UV irradiation. According to the TEM images and dynamic light scattering data, the photoswitching process involves self-assembly/disassembly from approximately $150 \mathrm{~nm}$ spherical particles to $18 \mathrm{~nm}$ irregular particles. We proposed a rational strategy using light-driven self-assembly for a molecular photoswitch in which optical chirality can be modulated by light irradiation in aqueous media.

\section{Received 13 October 2021; accepted 10 November 2021; published online 4 January 2022}

1 Moujaber O, Stochaj U. The cytoskeleton as regulator of cell signaling pathways. Trends Biochem Sci, 2020, 45: 96-107

2 Bandara YMNDY, Tang J, Saharia J, et al. Characterization of flagellar filaments and flagellin through optical microscopy and label-free nanopore responsiveness. Anal Chem, 2019, 91: 13665-13674

3 Zhang J, Wang X, Suo X, et al. Cellular response of Escherichia coli to photocatalysis: Flagellar assembly variation and beyond. ACS Nano, 2019, 13: 2004-2014

4 Cornelissen JJLM, Rowan AE, Nolte RJM, et al. Chiral architectures from macromolecular building blocks. Chem Rev, 2001, 101: 40394070

5 Hembury GA, Borovkov VV, Inoue Y. Chirality-sensing supramolecular systems. Chem Rev, 2008, 108: 1-73

6 Mendez-Arroyo J, d'Aquino AI, Chinen AB, et al. Reversible and selective encapsulation of dextromethorphan and $\beta$-estradiol using an asymmetric molecular capsule assembled via the weak-link approach. J Am Chem Soc, 2017, 139: 1368-1371

7 Qin $\mathrm{P}, \mathrm{Wu} \mathrm{Z}$, Li P, et al. Triple-modulated chiral inversion of coassembly system based on alanine amphiphile and cyanostilbene derivative. ACS Appl Mater Interfaces, 2021, 13: 18047-18055

8 Zheng G, He J, Kumar V, et al. Discrete metal nanoparticles with plasmonic chirality. Chem Soc Rev, 2021, 50: 3738-3754

9 Huo GF, Tu Q, Hu YX, et al. Amplified circularly polarized luminescence promoted by hierarchical self-assembly involving Pt...Pt interactions. Sci China Mater, 2022, 65:

10 Huang S, Yu H, Li Q. Supramolecular chirality transfer toward chiral aggregation: Asymmetric hierarchical self-assembly. Adv Sci, 2021, 8: 2002132

11 Xiao X, Pedersen SK, Aranda D, et al. Chirality amplified: Long, discrete helicene nanoribbons. J Am Chem Soc, 2021, 143: 983-991

12 Zhu H, Li Q, Gao Z, et al. Pillararene host-guest complexation induced chirality amplification: A new way to detect cryptochiral compounds. Angew Chem Int Ed, 2020, 59: 10868-10872

13 Bhanjadeo MM, Subudhi U. Praseodymium promotes B-Z transition in self-assembled DNA nanostructures. RSC Adv, 2019, 9: 4616-4620

14 Choi J, Majima T. Conformational changes of non-B DNA. Chem Soc Rev, 2011, 40: 5893-5909

15 Chen Z, Yin H, Wen Z, et al. Organic indoor light harvesters achieving recorded output power over $500 \%$ enhancement under thermal radiated illuminances. Sci Bull, 2021, 66: 1641-1648

16 Cong M, Yang B, Hong F, et al. Self-trapped exciton engineering for white-light emission in colloidal lead-free double perovskite nanocrystals. Sci Bull, 2020, 65: 1078-1084

17 Jerca FA, Jerca VV, Hoogenboom R. Photoresponsive polymers on the move. Chem, 2017, 3: 533-536

18 Li D, Zhu C, Liu H, et al. Light-triggered two-dimensional lateral homogeneous $\mathrm{p}-\mathrm{n}$ diodes for opto-electrical interconnection circuits. Sci Bull, 2020, 65: 293-299

19 Li M, Wang YF, Zhang DW, et al. Thermally activated delayed fluorescence material-sensitized helicene enantiomer-based OLEDs: A new strategy for improving the efficiency of circularly polarized electroluminescence. Sci China Mater, 2021, 64: 899-908

$20 \mathrm{Wu}$ Y, Guo Z, Zhu WH, et al. Photoswitching between black and colourless spectra exhibits resettable spatiotemporal logic. Mater Horiz, 2016, 3: 124-129

21 Jia C, Migliore A, Xin N, et al. Covalently bonded single-molecule 
junctions with stable and reversible photoswitched conductivity. Science, 2016, 352: 1443-1445

22 Chen X, Zhao W, Baryshnikov G, et al. Engineering stable radicals using photochromic triggers. Nat Commun, 2020, 11: 945

23 Wu NMW, Ng M, Yam VWW. Photochromic benzo[b]phosphole alkynylgold(I) complexes with mechanochromic property to serve as multistimuli-responsive materials. Angew Chem Int Ed, 2019, 58: 3027-3031

24 Zhang J, Zou Q, Tian H. Photochromic materials: More than meets the eye. Adv Mater, 2013, 25: 378-399

25 Qin L, Liu X, Yu Y. Soft actuators of liquid crystal polymers fueled by light from ultraviolet to near infrared. Adv Opt Mater, 2021, 9: 2001743

26 Marturano V, Bizzarro V, De Luise A, et al. Essential oils as solvents and core materials for the preparation of photo-responsive polymer nanocapsules. Nano Res, 2018, 11: 2783-2795

27 de Jong JJD, Lucas LN, Kellogg RM, et al. Reversible optical transcription of supramolecular chirality into molecular chirality. Science, 2004, 304: 278-281

28 Takaishi K, Yasui M, Ema T. Binaphthyl-bipyridyl cyclic dyads as a chiroptical switch. J Am Chem Soc, 2018, 140: 5334-5338

29 Zhang J, Zhou Y, Yao Y, et al. A light triggered optical and chiroptical switch based on a homochiral $\mathrm{Eu}_{2} \mathrm{~L}_{3}$ helicate. J Mater Chem C, 2020, 8: 6788-6796

30 Zhang L, Wang HX, Li S, et al. Supramolecular chiroptical switches. Chem Soc Rev, 2020, 49: 9095-9120

$31 \mathrm{Wu}$ Y, Xie Y, Zhang Q, et al. Quantitative photoswitching in bis(dithiazole)ethene enables modulation of light for encoding optical signals. Angew Chem Int Ed, 2014, 53: 2090-2094

32 Li M, Chen LJ, Cai Y, et al. Light-driven chiral switching of supramolecular metallacycles with photoreversibility. Chem, 2019, 5: 634648

33 Miao W, Wang S, Liu M. Reversible quadruple switching with optical, chiroptical, helicity, and macropattern in self-assembled spiropyran gels. Adv Funct Mater, 2017, 27: 1701368

34 Jin $\mathrm{X}$, Zhou M, Han J, et al. A new strategy to achieve enhanced upconverted circularly polarized luminescence in chiral perovskite nanocrystals. Nano Res, 2022, 15: 1047-1053

35 Cai Y, Guo Z, Chen J, et al. Enabling light work in helical self-assembly for dynamic amplification of chirality with photoreversibility. J Am Chem Soc, 2016, 138: 2219-2224

36 Chen S, Leung FKC, Stuart MCA, et al. Dynamic assemblies of molecular motor amphiphiles control macroscopic foam properties. J Am Chem Soc, 2020, 142: 10163-10172

37 Wu Z, Wang Q, Li P, et al. Photochromism of neutral spiropyran in the crystalline state at room temperature. J Mater Chem C, 2021, 9: 62906296

Acknowledgements This work was supported by the National Natural Science Foundation of China (21875143 and 21908146), the Innovation Research Foundation of Shenzhen (JCYJ20180507182229597), and the Natural Science Foundation of Guangdong Province (2016A030312002). Special thanks to the Instrumental Analysis of Shenzhen University (Lihu Campus).

Author contributions $\mathrm{Wu} \mathrm{Y}$ and $\mathrm{Yu} \mathrm{ZQ}$ conceived the research; Wu Y and Zeng $X$ designed the experiments; Zeng X, Zou L, Liu X and Qi X performed the experiments; Zeng $\mathrm{X}, \mathrm{Wu} \mathrm{Y}$ and $\mathrm{Yu} \mathrm{ZQ}$ analyzed the data; $\mathrm{Wu} \mathrm{Y}$ and Zeng $\mathrm{X}$ wrote the original draft; $\mathrm{Wu} \mathrm{Y}$ and $\mathrm{Yu} \mathrm{ZQ}$ completed the manuscript.

Conflict of interest The authors declare that they have no conflict of interest.

Supplementary information Experimental details and supporting data are available in the online version of the paper.

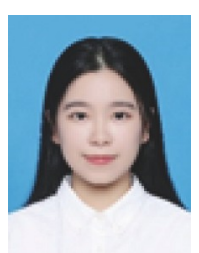

Xiaoxuan Zeng received her BSc degree from Huizhou University (2019). She is now an MSc student at the College of Chemistry and Environmental Engineering, Shenzhen University, under Prof. Zhen-Qiang Yu's supervision. Her research topic is chiral luminescent materials.

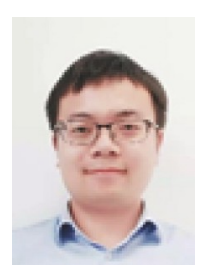

Yue Wu received his BSc degree from Jiangnan University and $\mathrm{PhD}$ degree from East China University of Science and Technology in 2014. Then he worked for WilmarArowana as a senior R\&D engineer for four years. In 2019 , he joined Shenzhen University. Currently, his research interests focus on chiral luminescence and photoswitch.

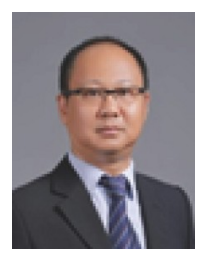

Zhen-Qiang Yu received his $\mathrm{BSc}$ and $\mathrm{PhD}$ degrees from the Department of Chemistry, Beijing Normal University in 1997 and the College of Chemistry, Peking University in 2007, respectively. He joined the College of Chemistry and Environmental Engineering, Shenzhen University in 2007. where he is currently an associate professor. His research interests are focused on the synthesis, structures, properties and applications of functional materials.

\section{水溶液介质中氰基苯乙烯衍生物的可逆手性光驱动 自组装}

曾晓璇, 吴玥", 邹林, 刘兴旺, 祁鍂, 余振强*

摘要 人工手性自组装一直是分子化学与材料化学的前沿挑战性课题. 但由于缺少合适的手性光响应结构单元，构造具有光控、可逆、手性 专一性响应的分子体系具有很大的挑战性. 本工作通过合理的分子设 计：以氰基二苯乙烯作为光致异构基团，磺酸基作为亲水基团，胆固醇 作为疏水手性基团, 构造了独特的光响应组装体系. 当氰基二苯乙烯处 于反式构型时, 在水相中组装形成规则的球状组装体且具有较强的手 性信号. 通过 $365 \mathrm{~nm}$ 光照后, 反式构型转变为顺式构型, 球状组装体解 组装, 同时手性信号消失; 当采用 $254 \mathrm{~nm}$ 光照后, 重新形成具有手性信 号的球状组装体. 核磁共振和吸收光谱结果表明光致异构转化率达到 $90 \%$ ，并且具有良好的光致异构重复性(抗疲劳性)及热稳定性. 本研究 成功实现了水相介质中光控、手性专一性响应的可逆光调控，在构建 双稳态光控手性开关方面表现出潜在的应用前景. 